\title{
RADIOFREQUENCY ABLATION IN THE TREATMENT OF LIVER METASTASES OF COLORECTAL CANCER
}

\author{
Adamov R. ${ }^{1}$, Mazyar F. ${ }^{2}$, Hamzik J. ${ }^{2}$ \\ ${ }^{1}$ Department of Surgery, Faculty Hospital, Zilina,Slovakia \\ ${ }^{2}$ Surgical Clinic, Jessenius Faculty of Medicine, Comenius University and University Hospital in Martin, \\ Slovakia
}

\begin{abstract}
A b s t r a c t
Many methods of minimally invasive therapy have been developed in recent years to eliminate tumours in organs like the liver. One of the most promising methods is called radiofrequency ablation, occasionally referred to as RFA. In radiofrequency ablation, a special needle electrode is placed in the tumour under the guidance of an imaging technique such as ultrasound (US), computed tomography (CT) scanning, or magnetic resonance (MR) imaging. A radiofrequency current is then passed through the electrode to heat the tumour tissue near the needle tip to cause tumour ablation. The heat from radiofrequency energy also closes up small blood vessels, thereby minimizing the risk of bleeding. In general, radiofrequency ablation causes only negligible discomfort and may be done as an outpatient procedure without general anaesthesia

In some cases radiofrequency ablation has made it possible to operate on patients that might not be good surgical candidates. Two types of liver tumour have been the main target of radiofrequency ablation; hepatocellular carcinoma, which is a primary liver cancer, and metastases from colorectal cancers to the liver. Many patients with a malignant liver tumour are not ideal surgical candidates as their tumours are so multifocal and inaccessible, that hepatectomy can lead to liver failure due to insufficient tissue reserve for adequate liver function. Other patients are poor surgical candidates due to poor performance status, as a consequence of other comorbidities, making major liver surgery exceedingly high risk. For many of these patients, radiofrequency ablation is a viable and effective treatment option.
\end{abstract}

Key words: radiofrequency ablation, minimally invasive therapy, liver tumour, colorectal liver metastases.

\section{INTRODUCTION}

The liver is a frequent target of primary and secondary malignant tumours. The most common liver malignancies are the hepatocellular carcinoma and metastases from the colorectal carcinomas. Hepatocellular carcinoma ranks fourth as the cause of death from malignant cancers worldwide. Approximately one million new cases of hepatocellular carcinomas are documented each year (1). If malignant tumours of the liver are not treated mortality can reach $100 \%$. The standard treatment option, in these tumours when appropriate is the surgical therapy that leads to significant survival benefits. In contrast, radiotherapy and chemotherapy alone or in combination have been not proven to be as beneficial for long-term survivals of these patients.

After lymph nodes, the most common site for the metastases of colorectal adenocarcinoma is the liver. Liver is also common target for metastasis from other types of cancers also. Surgical resection of the liver metastases in patients with colorectal adenocarcinoma in properly selected patients can lead to long-term survival interval in the range of $20 \%-35 \%$ (6). Nevertheless, only $5 \%$ to $15 \%$ of patients with primary or metastatic liver cancers, are deemed suitable for curative liver resection $(2,3)$. The limiting factor for such surgical therapy is the number of tumours, their size, location, and the degree of invasion into the vascular or biliary structures as well as the reduced remaining functional parenchyma postoperatively which can lead to hepatic failure especially in those with underlying cirrhosis.

Address for correspondence:

Robert Adamov, MD, Department of Surgery, Faculty Hospital, Vojtecha Spanyola 1739/43,

01001 Zilina, Slovakia

Phone:. +415110322; e-mail: robadamov@mail.t-com.sk 
5-year survival rate after successful surgical resection of the hepatocellular carcinoma with metastasis fluctuates in the range from $20 \%$ to $40 \%$ (2). Due to the high percentage of unresectable tumours of the liver the locoregional treatment is the only option in many cases (5). As an alternative to surgical therapy a number of minimally invasive procedures have been developed for patients with primary or metastatic liver cancer, who are not suitable surgical candidates. Procedures such as percutaneous ethanol injection (PEI), chemoembolization of liver tumours, radioembolization treatment and various other thermal ablation techniques compromise some of the most frequently used effective treatment modalities. PEI technique is mostly used to treat small hepatocellular carcinomas and is not usually applied for the treatment of metastatic liver disease. Chemoembolization is most frequently used for unresectable tumours $(2,4)$. In the field of thermal ablation there are two categories. One delivering extremely low-temperature to the target tissue leading to cryoablation, the other high temperature, leading to selective tissue necrosis using radiofrequency, focused ultrasound, microwave and laser for attainment of high temperature. Radiofrequency ablation (RFA) is a minimally invasive technique which has been utilized in cardiology, treatment of trigeminal neuralgia and osteoma for a very long time. Lately it has been applied with good results for the treatment of liver and lungs tumours. Even though the liver and the lungs are the most common place of use of the radiofrequency ablation, this technique is also used in the treatment of malignant tumours of kidneys, adrenal glands, breast and other malignant soft tissues tumours (7). In recent years, RFA is gaining greater popularity due to favourable results in the treatment of primary and secondary liver tumours. Recent studies point to a favourable rate of survival and effective local ablation effect (2).

\section{PATIENTS AND METHODS}

This study included patients with liver metastasis from colorectal carcinoma from January 2011 to February 2012. The patients were treated by hepatic surgical resection in combination with RFA. Resections were performed at the Department of Surgery FNsP Žilina and systematic chemo-radiotherapy was continued at the Department of Oncology of the same hospital. During the study, 19 hepatic resections were performed, 13 of which were for metastatic colorectal carcinoma. Of the total of 13 metastases of colorectal carcinoma, 11 were treated with hepatic resection alone and 2 underwent hepatic resection combined with RFA (15.3\%). Before embarking on surgical therapy all patients were histologically verified to have colorectal carcinomas and had their radiological staging by Computed Tomography (CT). Patients that underwent combined hepatic resection and radiofrequency ablation had both procedures performed concurrently during one operative session. All 13 patients were deemed eligible candidates for treatment of metastatic disease by hepatic resection or combined RFA and resections. Due to involvement of both lobes of liver and anatomic proximity of some of these metastatic lesions to main vessels, 2 patients were selected prospectively to undergo combined surgical and ablation therapy. Radiofrequency ablation was in both cases accomplished during the open procedure. This approach was preferred due to significant benefit achieved using intra operative ultrasound, which allows better and more accurate identification of the lesions, its size, location, and relation to vascular structures leading to better oncological control and also better preservation of healthy liver tissue. Perioperative US examination and Doppler US examination realised also in these patients. For the above RFA, Cool-Tip tm RF System RADIONICS - Model CC-1220VAC $50 \mathrm{~Hz}$ was used. The application and positioning of the Cool-Tip RFA needle and subsequently the process of ablation was guided with perioperative or intraoperative US. The process, in one of the patients, failed due to damage to the RFA needle, without the possibility of assessing the effectiveness of the therapy. A patient with problematic location of metastasis underwent RFA in hepatic segment $\mathrm{V}$ and resection of metastases in segments 
II and VIII. The surgical intervention was supplemented by axial transversostomy. Surveillance CT examination with a focus on the dynamics and character of changes in the liver after RFA was carried in the postoperative period, on the 5th day, after 2 months and 4 months. On the basis of the surveillance CT examinations the effectiveness of radiofrequency ablation was evaluated. The patients underwent the systematic chemo-radiotherapy, with a total dose of 50.4 Gy and concomitant application of Xeloda and monoclonal antibody therapy with Avastin. In the second phase of surgical treatment, laparoscopic resection of rectal adenocarcinoma was performed.

\section{RESULTS}

The aim of this study was the assessment of effectiveness and contribution of the RFA in combination with surgical resection in the management of patients with metastatic colorectal carcinoma. Patient with colorectal adenocarcinoma, who was designated to undergo combined ablative and hepatic resection as the first line treatment, underwent complex preoperative spiral CT scan and synchronous metastases in hepatic segments II, V and VIII were found. The size of the metastases in the 2nd segment was $17.4 \mathrm{~mm} \times 16.6 \mathrm{~mm}$ and in the 8th segment was $15.6 \mathrm{~mm} \times 15.4 \mathrm{~mm}$. Metastases in the 2nd and 8th segment were located on the surface, and appropriate to wedge resection. The size of the metastasis in the 5th hepatic segment was $30.2 \mathrm{~mm} \times 28.0 \mathrm{~mm}$. The metastatic lesions were in close proximity to the right branch of the portal vein and branches for hepatic segments V and VI, therefore RFA was indicated. Regular CT scans of the liver with dynamic phases were performed to assess the tumour status with a structured CT imaging protocol at day 5, 2 months and 4 months after the RFA. The patient was discharged from the hospital without complications on the 7th day postoperatively. Subsequently the patient underwent systematic chemotherapy in the frame of outpatient care.

\section{CT scan on the 5th day after the RFA}

Due to secondary inflammatory reaction of surrounding tissue, in the immediate postoperative period the ablation area increases progressively and the maximum change occurs on the 4 th or the 5th day postoperatively.

Under identical conditions, RFA generator creates a spherical shape of an ablation zone, about $7 \mathrm{~cm}$ in long-axis diameter. The size and shape of the ablation zone varies according to the type of RF device used, the local environment of hepatic tissue, the proximity of the vascular structures and type of tumour. On the 5th post-operative day characteristic symptoms after RFA of hepatic metastasis were presented. On the CT scan after complete ablation, the defect was at the centre of the tumour location, and was initially larger, from $30.2 \mathrm{~mm} \times 28.0 \mathrm{~mm}$ to $52.1 \mathrm{~mm} \times 29.1 \mathrm{~mm}$ (which ensures desired tumour-free margins). The interface was sharp, smooth, and devoid of nodularity. When CT contrast agents were used there was a complete lack of tumour enhancement, with thin, smooth rim enhancement at the interface (the interface between normal liver tissue and the ablation defect). The rim represented ablation-induced hyperaemia, and continued approximately 4 weeks after RFA. The finding usually persists for a few weeks, disappearing by 4 th weeks post operatively. 


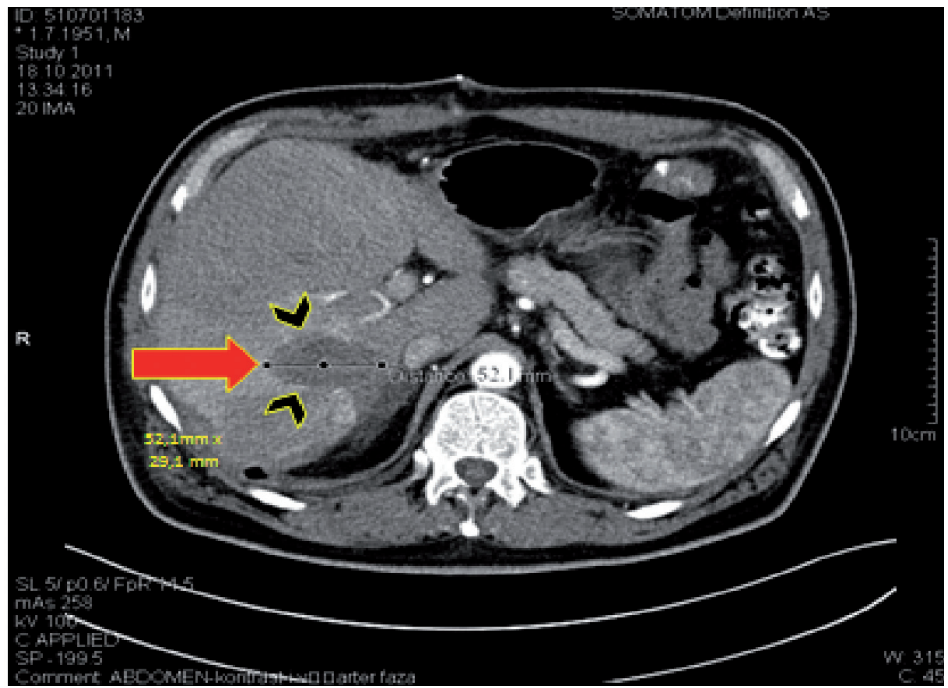

Fig. $1 \mathrm{CT}$ scan shows a thin enhancing rim due to ablation-induced hyperaemia. Note the thin smooth appearance and the lack of nodularity

\section{CT examination 2 months after the RFA}

The next CT scan confirmed complete destruction of metastases by occurrence

of hypovascularity. The next surveillance CT scan displayed that enlargement of the ablation area did not occur. An important indicator of successful ablation is stable size of the ablation defect or gradual decrease of the ablation area. A sudden increase in ablation defect is suspicious for the relapse of hepatic metastasis. In the case of relapse focal irregular area with extending size of the edge of lesion is frequently visualized on CT scans. A successfully treated tumour appears as a stable area of low density with the tendency of gradual shrinkage. Ablation area, which on subsequent surveillance CT displays as increase in size, is considered to be a local relapse. After 2 months no waistband of hyperaemia at the interface of the ablation area with liver tissue was observed. The edges of the ablation lesion were smooth, sharply bounded and the size of the ablation lesion was $52.3 \mathrm{~mm} \times 28.5 \mathrm{~mm}$.

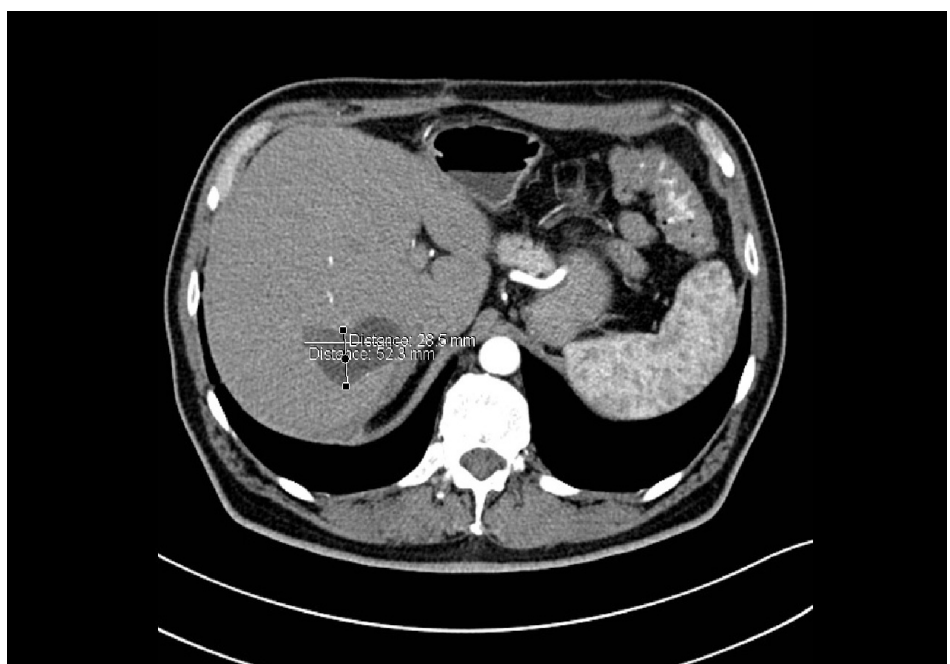

Fig. 2 CT scan 2 months after RFA with the image of the successful ablation of metastasis 


\section{CT examination 4 months after the RFA}

The last surveillance CT scan, before the second stage surgical therapy, was carried out 4 months after resection of metastases in segment II, VIII and after RFA of metastasis in segment V. During this period the patient underwent complex chemo radiotherapy. The CT scan displayed the area, of radiofrequency ablation as deposit with low density and a tendency to decrease in size without displaying irregular extensions to the size of the edge. The size of the deposit after ablation was 39.4 x $21.6 \mathrm{~mm}$ (Fig.3). In the area of rectal adenocarcinoma down staging was achieved.

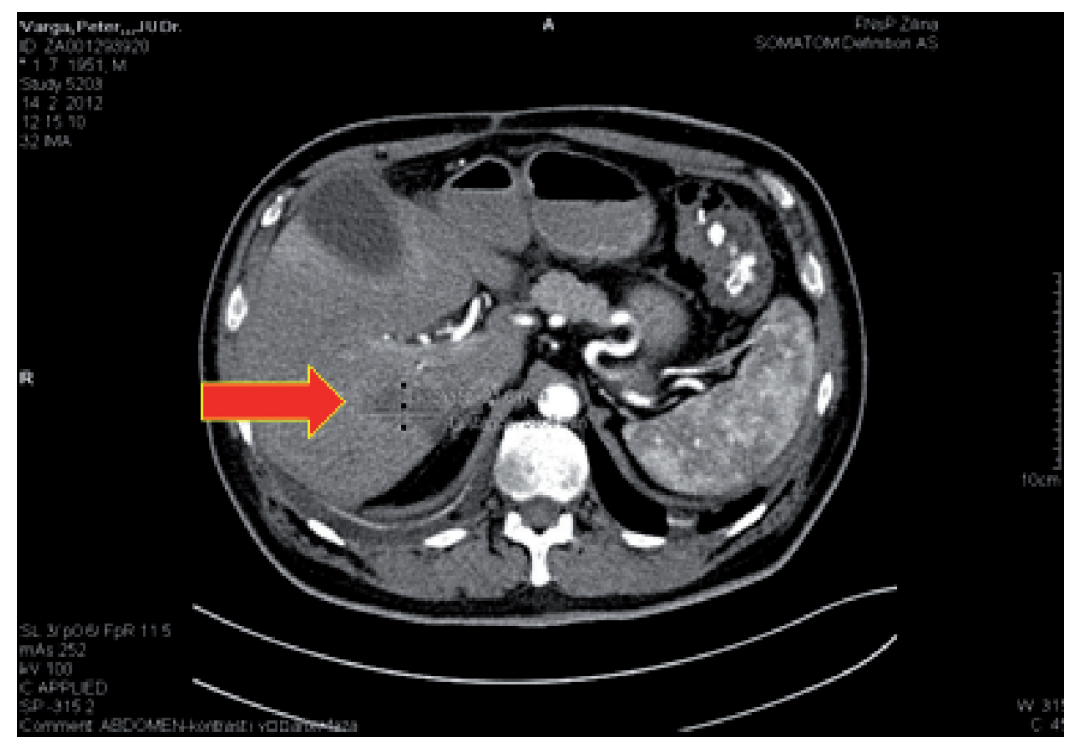

Fig. 3 CT 4 months after RFA, without image of metastatic relapse

Dynamic changes, using CT in the RF ablated area of metastasis in the 5th segment of liver were monitored for 4 months. The changes observed demonstrated a successful course of treatment, without complications and signs of progression or metastatic relapse (Table 1).

\begin{tabular}{|l|l|l|l|}
\hline $\begin{array}{l}\text { Metastaic } \\
\text { Lesion }\end{array}$ & 5th day after RFA & 2 months after RFA & 4 months after RFA \\
\hline Size & $52.1 \mathrm{~mm} \times 29.1 \mathrm{~mm}$ & $52 ., 3 \mathrm{~mm} \times 28.5 \mathrm{~mm}$ & $39.4 \mathrm{~mm} \times 21.6 \mathrm{~mm}$ \\
\hline Change of size & Increase in size & No change in size & Shrinkage in size \\
\hline Bounderies. & Hypereamic rim & $\begin{array}{l}\text { sharp, smooth, and } \\
\text { devoid of nodularity }\end{array}$ & Without progerssion \\
\hline & & & \\
\hline
\end{tabular}

Table 1 Postoperative monitoring of dynamic changes in the RFA area 
Localization of metastasis near the main vascular structures in the segment of right portal vein and the concurrent metastatic disease within the segment II of the left hepatic lobe made curative right hemihepatectomy unfeasible as the sole therapy, so segmental resection of metastasis in segment II was carried out, in combination with ablation of the other metastatic lesion by RFA. This method brought about surgical desired effect and subsequently allowed surgical resection of colorectal cancer to be carried out at a later date.

\section{DISCUSSION}

Anatomic or partial resection are established therapies for patients with liver_tumours. In patients with liver metastases from colorectal cancer, partial hepatectomy is a potentially curative treatment in about $30-50 \%$ of patients (8). While in the early days of surgical therapy for colorectal liver metastases the criteria for surgical resection was quite strict. Gradually new guidelines were adopted that extended indication criteria for partial liver resection in metastatic colorectal cancers, as it was shown that the procedure improves survival and at times leads to cure. With the expansion of criteria for surgical resection the limits of hepatic surgery were attained and additional procedures became necessary to render patients' tumour free. Thermoablation is one of these advanced techniques, which is being used with increased frequency in recent times. RFA is a technique which is based on the conversion of electromagnetic energy into heat. It is used for the destruction of tumour tissue. Ablative technique is often used in combination with partial liver resection and provides the possibility of treatment for patients with liver tumours, who cannot be cured by partial liver resection. Many newly diagnosed liver tumours are surgically unresectable. However, in selected cases radiofrequency ablation seems to be the only option. The factors limiting feasibility of surgical resection are the tumour size, the total number, location of tumours and its relation to main blood vessel and other vital structures. Radical surgical therapy can leads to the reduced liver function reserve which can be devastating, especially in patients with underlying liver disease such as cirrhosis. Radiofrequency ablation in patients who are not suitable candidates for surgical therapy, offers a minimally invasive therapeutic technique with low level of mortality and serious complications. It is suitable option that can be considered in patients that have resectable metastatic disease but one or more of the metastatic lesions are impossible or difficult to safely resect due to its anatomical proximity to vasculature. In these patients surgical resection may be combined with RFA. RFA cannot replace surgical resection, which should remain as the gold standard in the treatment of malignant liver tumours. However, it should be considered in cases where surgical resection leads to considerable loss of hepatic reserve, or when surgical resection is not feasible due to anatomic positions of tumour. In appropriate situation it can be utilized as sole therapy or in combination with surgical resection. The aim of this study was to assess the feasibility and efficiency of combined hepatic resection and RFA in the treatment of colorectal liver metastases. Despite many positive theoretical benefits about the influence of the combined therapy, there are also some doubts in the surgical community, as the ablative therapy combined with wide surgical resection is considered unsafe and thought to complicate an already demanding surgical resection (9). Moreover, there is an assumption that patients who are candidates for surgical resection represent a group with a unfavourable prognosis because of the high risk disease. Obviously the application of combined resection and RFA, which increases the operative risk and also postoperative complication is unfounded, if it does not brings positive results in term of increasing the long-term survival (9). According to other studies the complication rate for combined hepatic resection and RFA was $19,8 \%$, which is comparable to the $11 \%$ to $35 \%$ morbidity rates reported for hepatic resection alone (9). During the study period, there were no complications neither during the operation nor during the observation period after the surgical treatment of colorectal liver metastases. Ablative destruction of the problematic 
metastasis in segment $\mathrm{V}$ was realized without damaging the right branch of portal vein and branches for segment V and VI, which were located in close proximity to the liver metastasis. In general, RFA combined with liver resection is regarded as a safe and well tolerated method of surgical therapy. However, in some studies $2.3 \%$ mortality after combined surgical therapy is reported and this points out the seriousness of the radical surgical therapy of malignant liver tumours (9). Although, the combined surgical therapy seems to be safe in most cases liver resection combined with thermal destruction of extensive liver volume may lead to liver failure and to death. Patients with liver cirrhosis with reduced hepatic reserve and also patients' with normal liver that have or should undergo extensive liver resection and RFA must be properly assessed for risk of hepatic failure and selected accordingly. Malignancies of the liver must be properly assessed in relation to the volume of remaining functional hepatic parenchyma. In a recent systematic review of reported series on the combination of RFA with liver resection 3- and 5-year survival rates of 45 and 30\% for patients with colorectal liver metastases were reported (10). Patients with rectal carcinoma presenting with simultaneous liver metastases, who are treated with surgical resection combined with RFA represent a special category from an oncosurgical point of view. Multiple metastases, bi-lobar lesions or relapse after previous liver resection gives these patient poor prognosis . Despite these adverse factors survival results are comparable with the results of survival in patients with colorectal metastases treated with partial liver resection. Clinical studies have shown that surgical resection alone or in conjunction with RFA therapy leads to improved survival rate in comparison with chemotherapy alone.

\section{REFERENCES}

1. Lau, W.Y. Primary hepatocellular carcinoma. In: Blumgart, L.H., Fong, Y, eds. Surgery of liver and biliary tract. Vol II. 3rd ed. London, UK: WB Saunders; 2000:1423-1450.

2. Krishnamurthy, V.N., Casillas, V.J., Latorre, L. Radiofrequency Ablation of Hepatic Lesions: A Review : Applied Radiology. 2003, vol.32(10) : 11-26.

3. Framer, D.G., Rosove, M.H., Shaked, A. , et al. Current treatment modalities for hepatocellular carcinomas. Annals of Surgery. 1994, vol.219(3) : 236-247.

4. Clouse, M.E., Lee, R.G., Duszlak, E.J., et al. Peripheral hepatic artery embolization for primary and secondary hepatic neoplasms. Radiology. 1983, vol.147(2) : 407-411.

5. Rhim H, Lim H.K, Kim Y.S, Choi D, Lee W.J. Radiofrequency Ablation of Hepatic Tumors: Lessons Learned From 3000 Procedures. Journal of Gastroenterology and Hepatology. 2008, vol.23(10) : 1492-1500.

6. Curley, S.A, Izzo, F., Delrio, P., Ellis, L.M., Granchi, J., Vallone, P., Fiore, F., Pignata, S., Daniele, B., Cremona, F. Radiofrequency Ablation of Unresectable Primary and Metastatic Hepatic Malignancies. Results in 123 Patients. Annals of Surgery. 1999, vol.230(1) : 1.

7. Feliberti, E.C, Wagman, L.D. Radiofrequency Ablation of Liver Metastases from Colorectal Cancer. Cancer Control. 2006, vol.13(1) : 48-51.

8. Koert P. de Jong, Marieke W.J.L.A.E. Wertenbroek. Liver Resection Combined with Local Ablation: Where Are the Limits? Digestive Surgery. 2011, vol.28(2):127-133.

9. Timothy M. Pawlik, Francesco Izzo, Deborah S. Cohen, Jeffery S. Morris, Steven A. Curley. Combined Resection and Radiofrequency Ablation for Advanced Hepatic Malignancies: Results in 172 Patients. Annals of Surgical Oncology. 2003, vol.10(9) : 1059-1069.

10. Eisele RM, Zhukowa J, Chopra S, Schmidt SC, Neumann U, Pratschke J, Schumacher G: Results of liver resection in combination with radiofrequency ablation for hepatic malignancies.Eur J Surg Oncol 2010, vol.36(3) : 269-274.

Received: July 11,2012

Accepted: August 29,2012 